\title{
Validity of the fractional Leibniz rule on a coarse-grained medium yields a modified fractional chain rule
}

\author{
J. Weberszpil \\ UFRRJ - Instituto Multidisciplinar \\ Depto. Tecnologias e Linguagens \\ Nova Iguaçú, RJ \\ E-mail: josewebe@gmail.com.
}

\begin{abstract}
In this short communication, we show that the validity of the Leibniz rule for a fractional derivative on a coarse-grained medium brings about a modified chain rule, in agreement with alternative versions of fractional calculus. We compare our results to those of a recent article on this matter.
\end{abstract}

Keywords: fractional Leibniz rule, fractional chain rule, Hölder Space, coarse-grained medium, nondifferentiable functions

\section{Introduction}

Fractional calculus (FC) is a mathematical tool which has a wide range of applications to mathematical modelling in physical sciences, biological and biomedical engineering, control systems and dynamic modelling of soils, among many others $[18,7]$. It has a history of over 300 years and an extensive bibliography has been produced on this subject matter. Complex systems whose dynamical behaviour is described by so-called anomalous functions, with fractional powers decreasing behaviour are well described by the FC machinery. There are several approaches to FC, depending on the applications envisaged.

In a recent article [17], it is argued that violation of the Leibniz rule is a characteristic property of non-integer order derivatives, and that non-integer order derivatives satisfying the Leibniz rule must be of entire order $\alpha=1$. In the same article, the author suggests that the result, which has been proven for a function $f$ of class $C^{2}, f \in C^{2}(U)$, would hold also for any formulation of FC applied to functions that are not necessarily differentiable.

In certain physical models of our real world, one deals with observables that are not necessarily differentiable functions, and may have fractal characteristics (so-called 'coarse-grained' spaces [6]), see e.g. [15], [13] or [2].

A continuous, nowhere integer-differentiable function, necessarily exhibits random-like or pseudo-random features, which links this story to extensions of the theory of stochastic differential equations to describe stochastic dynamics driven by fractional Brownian motion $[8,9,10]$. For an interesting effort in building up a solid geometry and field theory in fractional spaces, see $[2,3,4,5]$.

A good mathematical framework for fractional derivative operators is that of Hölder spaces $H^{\lambda}$ [14], which finds abundant applications even in the stock market [16].

In this letter, we follow in the footsteps of [17] and start with a fractional differential operator $D^{\alpha}$ of order $\alpha$, which is assumed to satisfy the Leibniz rule, then derive a fractional chain rule of sorts for $D^{\alpha}$. Throughout we deal with identities just as in [17], and not with $L^{2}$-type almost-everywhere equalities throughout. 


\section{Hölder space and coarse-grained media}

According to Samko et al. [14], let $\Omega=(a, b),-\infty<a<b<\infty$, so $\Omega$ may be a finite interval, a half-line or the whole line. First let $\Omega$ be a finite interval. A function $F(x)$ is said to satisfy the Hölder condition of order $\lambda$ on $\Omega$ if

$$
\left|F\left(x_{1}\right)-F\left(x_{2}\right)\right| \leq A\left|x_{1}-x_{2}\right|^{\alpha},
$$

for any $x_{1}, x_{2} \in \Omega$, where $A$ is a constant and $\alpha$ is the Hölder exponent. If the function $f(x)$ satisfies the Hölder condition it is continuous on $\Omega$ [14], that is, if the Hölder coefficient is merely bounded on compact subsets of $\Omega$, then the function $F$ is said to be locally Hölder-continuous with exponent $\alpha$ in $\Omega$.

Now to define the Hölder space, let again $\Omega$ be a finite interval. We denote by $H^{\lambda}=H^{\lambda}(\Omega)$ the space of all functions which in general are complex valued, and satisfying the Hölder condition of a fixed order $\lambda$ on $\Omega$. This is a locally convex topological vector space.

Hölder space and nowhere differentiable functions are related. An immediate example of this is the Weierstrass function that is nowhere integer-differentiable [11]. The Weierstrass function may be written as

$$
W_{\alpha}(x)=\sum_{n=0}^{\infty} b^{-n \alpha} \cos \left(b^{n} x\right)
$$

for some $0<\alpha<1$. Then $W_{\alpha}(x)$ is Hölder-continuous of exponent $\alpha$, which is to say that there is a constant $\mathrm{C}$ such that

$$
\left|W_{\alpha}(x)-W_{\alpha}(y)\right| \leq C|x-y|^{\alpha}
$$

for all $\mathrm{x}$ and $\mathrm{y}$. Moreover, $W_{1}$ is Hölder-continuous of all orders $\alpha<1$ but not Lipschitz continuous.

\section{Main results}

Below we derive, out of the Leibniz rule, a fractional chain rule of sorts for compositions $f \circ w$ where $f$ is $C^{2}$ and $w$ is not necessarily $C^{1}$, similarly as in [7]. Here, we assume the same premisses for the conditions of operational linearity, the fractional derivative of a constant being zero and the validity of the fractional Leibniz rule as in [17].

Just as a locally $L^{1}$ function $f$ is a measurable function which is $L^{1}$ at every compact interval, we call a function locally Hölder of exponent $\alpha$ if and only if $f$ is Hölder of exponent $\alpha$ on every finite interval $[a, b]$. One such example is that of $f \in C^{1}(\mathbb{R})$, taking $\alpha=1$. Let $f, g$ be functions that are locally Hölder-continuous of exponent $\alpha$, namely, satisfying the Hölder condition of exponent $\alpha$ on every compact interval $[a, b]$ of their domain. Then so is $f g$.

Proof. It is entirely analogous to that of the formula for the derivative of a product. $\square$ Let $f \in C^{2}(I)$, where $I, J \subset \mathbb{R}$, and let $w: J \rightarrow I$ be a Hölder-continuous function of exponent $0<\alpha<1$, where $J \subset \mathbb{R}$ is another interval. Consider a fractional derivative $D^{\alpha}$ of order $\alpha$, satisfying the Leibniz rule, whose domain includes all locally Hölder-continuous functions of order $\alpha$. Then the following statement holds:

$$
D_{x}^{\alpha}(f \circ w)=D_{w}^{\alpha} f(w(x))\left(D_{x}^{\alpha} w(x)\right) .
$$

Proof. Clearly, $D_{x}^{\alpha} 1=0$ [17], since the Leibniz rule is assumed. Let $x_{0} \in J$, and let $t_{0}=w\left(x_{0}\right)$ and $t=w(x)$. Indeed, by repeated integration by parts one obtains the following identity:

$$
f(t)-f\left(t_{0}\right)=f^{\prime}\left(t_{0}\right)\left(t-t_{0}\right)+g_{2}(t)\left(t-t_{0}\right)^{2},
$$


where $g_{2}(t)=\int_{0}^{1}(1-s) f^{\prime \prime}\left(t_{0}+s\left(t-t_{0}\right)\right) d s$. Note that,

$$
g_{2}(t)=\left\{\begin{array}{ccc}
\frac{f(t)-f\left(t_{0}\right)}{\left(t-t_{0}\right)^{2}}-\frac{f^{\prime}\left(t_{0}\right)}{\left(t-t_{0}\right)}=\int_{t_{0}}^{t} \frac{(t-\tau) f^{\prime \prime}(\tau)}{\left(t-t_{0}\right)^{2}} d \tau, & t \neq t_{0} \\
2^{-1} f^{\prime \prime}(x), & t=t_{0}
\end{array},\right.
$$

and using Taylor's theorem

$$
g_{2}(t)=\left\{\begin{array}{cc}
\frac{2^{-1} f^{\prime \prime}\left(t_{0}\right)\left(t-t_{0}\right)^{2}+o\left(\left(t-t_{0}\right)^{2}\right)}{\left(t-t_{0}\right)^{2}}, & t \neq t_{0} \\
2^{-1} f^{\prime \prime}(x), & t=t_{0}
\end{array}\right.
$$

From which it follows directly that $g_{2}(t)\left(t-t_{0}\right)$ and $g_{2}(t)\left(t-t_{0}\right)^{2}$ belong, respectiely, to $C^{1}(I)$ and $C^{2}(I)$.

Now, from eq.(5) one obtains the following identity:

$$
f(w(x))=f\left(w\left(x_{0}\right)\right)+f^{\prime}\left(w\left(x_{0}\right)\right)\left(w(x)-w\left(x_{0}\right)\right)+\left[g_{2}(w(x))\left(w(x)-w\left(x_{0}\right)\right)\right]\left[w(x)-w\left(x_{0}\right)\right] .
$$

Applying the Leibniz rule to the product $\left[g_{2}(w(t))\left(w(t)-w\left(x_{0}\right)\right)\right] \cdot\left(w(x)-w\left(x_{0}\right)\right.$ yields

$$
D_{x}^{\alpha}\left(\left[g_{2}(w(x))\left(w(x)-w\left(x_{0}\right)\right)\right] \cdot\left(w(x)-w\left(x_{0}\right)\right)=\left(w(x)-w\left(x_{0}\right)\right) H(x),\right.
$$

for a suitable function $H(x)$. It follows that, for $x=x_{0}$,

$$
\left.D_{x}^{\alpha}\left(g_{2}(w(x))\left(w(x)-w\left(x_{0}\right)\right)^{2}\right)\right|_{x=x_{0}}=0 .
$$

Thus, on applying $D_{x}^{\alpha}$ to (8) and evaluating on $x_{0} \in J$, one obtains

$$
D_{x}^{\alpha}(f \circ w)\left(x_{0}\right)=f^{\prime}\left(w\left(x_{0}\right)\right)\left(D_{x}^{\alpha} w\right)\left(x_{0}\right),
$$

which settles the Theorem. We remark that every function $w$ in the domain of $D^{\alpha}$ is allowed in our formula, provided $f \circ w$ also lies in its domain.

\section{Non-Integer Differentiable Functions}

Consider now that $w$ is of class $C^{1}$ and $f$ is Hölder-continuous, then for Riemann-Liouville fractional derivatives and even for the Caputo definition, the scale property holds through

$$
D_{x}^{\alpha} f(\lambda x)=\lambda^{\alpha} D_{w}^{\alpha} f(w) ; \quad w=\lambda x .
$$

But, considering the relation

$$
\lambda^{\alpha}=\left[D_{x}^{1}(\lambda x)\right]^{\alpha}
$$

then, eq.(12) can be rewritten as

$$
D_{x}^{\alpha} f(\lambda x)=\left[D_{x}^{1}(\lambda x)\right]^{\alpha} D_{w}^{\alpha} f(w)
$$

or

$$
D_{x}^{\alpha}(f \circ w)=\left[\left(D_{w}^{\alpha} f\right) \circ w\right] \cdot\left(w^{\prime}\right)^{\alpha} .
$$

Eq. (15) turns out to be the same chain rule that is valid for nondifferentiable functions in the alternative versions of FC [7], considering $w=\lambda x$ as differentiable while $f(w)$ is nondifferentiable. 


\section{Conclusions}

Departing from the main result in [17], we endeavoured to study the case where the fractional derivative $D_{x}^{\alpha}$ contains locally Hölder functions of exponent $\alpha$ in its domain, and satisfies the Leibniz rule. Hadamard's representation theorem for $f \in C^{2}$ around an arbitrary point yields a fractional chain rule for such $D^{\alpha}$ applied to $f \circ w$, where $w$ is locally Hölder of exponent $\alpha$, cf. eq (11). As well, we have seen in (14) a formula in the case where $w$ is of class $C^{1}$ and $f$ is Hölder-continuous.

Our main goal here was to reassess a generalization of the Main Theorem in [17]. In so doing, we derived a fractional chain rule for a fractional derivative of order $0<\alpha \leq 1$ whose domain includes $C^{1}$ or even locally Hölder-continuous functions (which describe coarse-grained media), provided the Leibniz rule holds. This in turn implies that Leibniz role holds for some alternative definitions of fractional derivatives $[7,11,1,12]$.

Acknowledgment: J. A. Helayël-Neto and J. J. Ramón-Marí are gratefully acknowledged for discussions at all stages of this work.

\section{References}

[1] Faál Ben Adda and Jacky Cresson, "About Non-differentiable Functions", Journal of Mathematical Analysis and Applications 263, (2001) 721-737.

[2] G. Calcagni, A. Eichhorn, F. Saueressig, Phys. Rev. D 87, (2013) 124028.

[3] G. Calcagni, Adv. Theor. Math. Phys. 16 (2012) 549-644; arXiv:1106.5787.

[4] G. Calcagni, JHEP01(2012)065; arXiv:1107.5041.

[5] Gianluca Calcagni, João Magueijo, and David Rodríguez Fernández, arXiv:1305.3497.

[6] Gell-Mann, Murray and Hartle, James B., "Quasiclassical coarse graining and thermodynamic entropy", Phys. Rev. A 76, (2007) 022104.

[7] Guy Jumarie, "On the derivative chain-rules in fractional calculus via fractional difference and their application to systems modeling, Cent. Eur. J. Phys. (2013) 1-17; Jumarie G., J. Appl. Math. \& Computing Vol. 24, No. 1 - 2, (2007) 31 - 48; Applied Mathematics Letters 22, (2009) 378-385;. Guy Jumarie, "Table of some basic fractional calculus formula derived from a modified Riemann-Liouville derivative for nondifferentiable functions", Applied Mathematics Letters 22 (2009) 378-385.

[8] Guy Jumarie, J. Appl. Math. \& Computing 24, No. 1-2, (2007) 31-48; Applied Mathematics Letters 22, (2009) 378-385; Applied Mathematics Letters 22, (2009) 378-385.

[9] G. Jumarie, Chaos, Solitons and Fractals 41, (2009) 1590-1604.

[10] G. Jumarie, Computers \& Mathematics with Applications 59(3) (2010) 1142-1164.

[11] Kiran M. Kolwankar and Anil D. Gangal,"Fractional differentiability of nowhere differentiable functions and dimensions", Chaos 6 (1996), 505.

[12] K. M. Kolwankar, J. J. Lévy Véhel ,"Measuring functions smoothness with local fractional derivatives", FCAA 4, 3 (2001) 285-301.

[13] L. Nottale Fractal space-time and micro physics. Singapore: World Scientific; 1993; Chaos Solitons Fract 4 (1994) 361-388. 
[14] S.G. Samko, A.A. Kilbas and O.I. Marichev, Fractional integrals and derivatives, Gordon and Breach, New York, USA, 1993.

[15] F.H. Stillinger, J. Math. Phy. 18 (1977) 1224.

[16] Yu. A Kuperin, R.R. Schastlivtsev, "Modified Hölder Exponents Approach to Prediction of the USA Stock Market Critical Points and Crashes", arXiv:0802.4460.

[17] Tarasov, V.E. "No violation of the Leibniz rule. No fractional derivative", Commun. Nonlinear Sci. Numer. Simulat. 18, (2013) 2945-2948.

[18] J. Weberszpil, C.F.L. Godinho, A. Cherman and J.A. Helayël-Neto, " Aspects of the CoarseGrained-Based Approach to a Low-Relativistic Fractional Schrödinger Equation", In: 7th Conference Mathematical Methods in Physics - ICMP 2012, 2012, Rio de Janeiro. Proceedings of Science (PoS). Trieste, Italia: SISSA. Trieste, Italia: Published by Proceedings of Science (PoS), 2012. p. 1-19; C.F.L. Godinho, J. Weberszpil, J.A. Helayël-Neto, "Extending the D'Alembert Solution to Space-Time Modified Riemann-Liouville Fractional Wave Equations", Chaos, Solitons \& Fractals 45, (2012) 765-771. 